\title{
DIETA Y USO DE ANIMALES EN EL POTOSÍ COLONIAL
}

\author{
DIET AND ANIMAL USE IN COLONIAL POTOSÍ
}

\author{
Susan D. deFrance ${ }^{1}$
}

\begin{abstract}
La colonización española de la región de Potosí, Bolivia, fue acompañada por cambios en la producción de alimentos, su distribución y en las prácticas locales de consumo. Los españoles establecieron animales de origen euroasiático para crear una fuente de carne que era familiar y aceptable a la práctica ibérica tradicional. El grado de cambio dependió del sistema existente de producción de alimento, la localización geográfica del establecimiento colonial, la composición demográfica de la colonia y los sistemas de comercio. En el Alto Perú, Potosí, la fusión colonial de animales euroasiáticos y de recursos andinos resultó en el establecimiento de prácticas que fueron distintas en las Américas. Las excavaciones de sitios coloniales en Porco demuestran que los trabajadores indígenas adoptaron pocos animales de origen euroasiático. Un análisis zooarqueológico indica que la mayoría de los restos de fauna son de origen local. El patrón de uso de animales en Porco contrasta con el patrón documentado en el sitio colonial de Tarapaya donde los residentes españoles superaron obstáculos de distancia, el deterioro de los alimentos y de transporte para adquirir animales de origen euroasiático así como una gama muy diversa de productos comestibles incluyendo una variedad de pescados.

Palabras claves: zooarqueología, Potosí, animales euroasiáticos, colonización española.
\end{abstract}

Spanish colonization in the region of Potosí, Bolivia was accompanied by changes in local food production, distribution, and consumption practices. Spaniards established animals that were of Eurasian origin to create a supply of meat that was familiar and acceptable to traditional Iberian practice. The degree of change was dependent on the existing food production system, the geographic location of the colonial settlement, the size and demographic composition of the colony, and trade and exchange systems. In Upper Peru, the colonial fusion of Eurasian animals and Andean resources resulted in the emergence of colonial practices that were distinct in the Americas. Excavations of colonial sites in Porco demonstrate that the indigenous workers who resided in the mining communities adopted relatively few animals of Eurasian origin. A zooarchaeological analysis indicates that the majority of the fauna is of local origin. The pattern of animal use at Porco is in stark contrast to the pattern of animal use documented at the colonial site of Tarapaya where Spanish residents overcame obstacles of distance, spoilage, and transport to acquire animals of Eurasian origin as well as a very diverse range of foodstuffs including a variety of fish.

Key words: Zooarchaeology, Potosí, Eurasian animals, Spanish colonization.

El registro arqueológico del Potosí Colonial es único a través de todo el Imperio Español debido a su escala monumental de ciudad y a la diversidad cosmopolita de sus habitantes. Personas de varios niveles étnicos y socioeconómicos habitaron en el centro urbano y regiones circundantes. Como la más grande ciudad colonial en las Américas, la economía creciente fomentó la creación y transformación de identidades culturales y las condiciones materiales de la vida colonial. La dieta y los modos alimenticios son aspectos de la cultura que pueden ser perdurables (i.e. resistentes al cambio o resistentes en los tiempos de cambio) y también pueden estar sujetos a las innovaciones o cambios. Los modos alimenticios que se desarrollaron en los asentamientos coloniales reflejan la combinación de los hábitos alimenticios existentes y los animales introducidos junto con métodos culturalmente prohibidos de adquisición, preparación y consumo de alimentos. Las reglas culturales para la adopción de ciertos animales como alimento fueron probablemente más fuertemente sancionadas que las reglas para la adopción de animales con fines utilitarios. Las condiciones del medio ambiente local también establecieron restricciones en el éxito de la reproducción y supervivencia de los animales.

La industria minera utilizó los animales y sus productos tanto para alimento como para una variedad de fines económicos. Es por esto que el material arqueológico asociado con los sitios mineros en las Américas proporciona una oportunidad única para determinar el grado en que las poblaciones adoptaron animales de origen foráneo, así como el grado de éxito que se experimentó con la introducción de estos animales. El medio ambiente proporcionó los parámetros para la supervivencia de los animales sobre los cuales la gente podría imponer una selección

$\overline{1}$ Department of Anthropology, PO Box 117305, University of Florida, Gainesville, Fl, 32611. sdef@ufl.edu 
cultural para su uso alimenticio. De otro lado, los animales y sus restos pueden también ser usados para identificar rutas de intercambio y movimientos comerciales de animales comestibles.

Hasta ahora, las excavaciones realizadas en dos sitios coloniales cerca de Potosí han producido gran cantidad de restos zooarqueológicos (Figura 1). Hacia el sur de Potosí, cerca del pueblo moderno de Porco, los investigadores han recuperado restos de fauna procedentes de las excavaciones en el sitio doméstico de Cruz Pampa, ubicado al sudeste de Porco. En este artículo se compara el corpus de fauna de Cruz Pampa con el del sitio de Tarapaya, un sitio de hospedaje y residencia colonial, ubicado al norte de Potosí. Ambos sitios son contemporáneos y datan de la primera mitad del siglo XVII (Eighmy et al. 2006). Las excavaciones fueron dirigidas por Mary Van Buren (Van Buren 1999). Estos dos sitios y sus habitantes asociados difieren en lo referente al estatus social y etnicidad; también difieren en ubicación geográfica, elevación y función del sitio. De esta manera, las comparaciones de los restos de fauna representan un gran potencial para documentar los usos diferenciales de los animales durante el período colonial.

Como se muestra en este capítulo, los trabajadores indígenas que vivían en Cruz Pampa mantuvieron una dieta de carácter muy andino con el uso de unos pocos animales introducidos. En contraste, los españoles de alto estatus que habitaron en Tarapaya fueron capaces de superar los obstáculos de distancia, transporte y elevación a fin de obtener animales comestibles exóticos y recursos ibéricos de carne. A pesar que los dos sitios están ubicados en diferentes áreas geográficas y que Tarapaya se encuentra en una elevación más baja (3.300 msm versus $4.100 \mathrm{msm}$ ), se sostiene que el estatus socioeconómico y las diferencias étnicas fueron probablemente las mayores determinantes en las prácticas de consumo de carne que la elevación, asentamiento o función del sitio. Antes de describir los materiales zooarqueológicos en detalle, se presenta un resumen de los aspectos del uso y adquisición de animales durante el período colonial.

En los últimos años la arqueología histórica se ha ampliado considerablemente en cuanto a su ámbito geográfico y teórico de investigación (ver Funari et al. 1999; Funari et al. 2005; Hall y Silliman 2006). Este estudio de caso contribuye al conocimiento sobre la subsistencia durante la colonia española en los Andes Centrales, y también al estudio de la arqueología histórica en América del Sur en general. La combinación de información histórica y zooarqueológica sirve como modelo para estudios futuros. En la medida que se hagan más estudios por parte de otros investigadores en historia de fauna de los Andes y otros lugares de América del Sur, podremos entender la variedad de experiencias coloniales españolas en todo el continente.

\section{Uso de los Animales durante el Período Colonial}

Los colonos españoles introdujeron animales euroasiáticos para proporcionar carne, productos animales y para el transporte. En los Andes Centrales, los españoles encontraron un medio ambiente menos húmedo y más montañoso que en otras áreas neotrópicas de asentamientos hispanos. Ésta fue también la única área en las Américas con una creciente economía prehispánica de pastoreo donde la gente crió mamíferos domesticados (llamas y alpacas) para una variedad de fines, sin embargo, la alta elevación de los asentamientos presentó otros cambios para la fauna introducida. Existieron muchos efectos negativos en los animales no nativos producidos por la altura y condiciones hipóxicas; dentro de éstos, las pérdidas fueron comunes. Los animales presentaban falta de peso y a menudo no sobrevivían. Muchos animales euroasiáticos estaban adaptados para parir en la noche; sin embargo, esto fue en detrimento de su descendencia debido a la fuerte disminución de la temperatura nocturna en la altura. Debido a que los pastizales alrededor de Potosí fueron escasos para los animales no nativos (p.ej., ovejas, cabras, vacas, caballos, burros), éstos fueron a menudo criados en elevaciones más bajas (Jiménez de la Espada 1965 [1603], Vol I).

Las grandes empresas mineras y otras actividades económicas del Potosí colonial eclipsaron la economía de pastoreo. Las altas elevaciones (>3.600 msm), asentamiento de muchas de las actividades mineras, fueron inhóspitas para la crianza de animales no nativos. A pesar que los sistemas agrícolas y de pastoreo andinos fueron notables por su adaptabilidad a los hábitats de altura del altiplano o las altas mesetas del hábitat de puna, Potosí probó ser resistente aun a las variedades más fuertes de plantas y animales indígenas. José de Acosta (2002 [1590]:172), impresionado por el clima seco y 


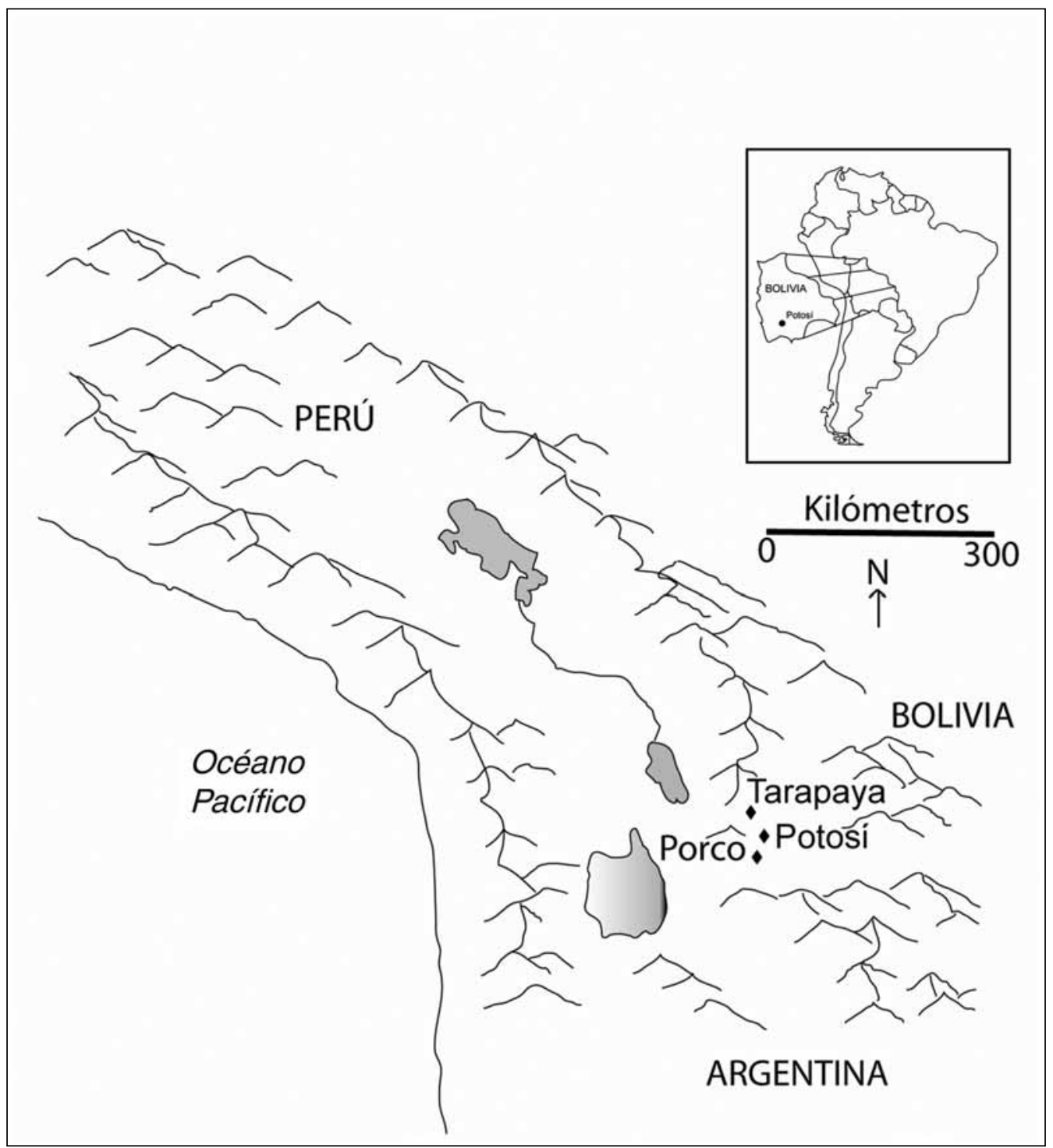

Figura 1. Mapa de ubicación de Porco y Tarapaya, Bolivia. Location map of Porco and Tarapaya, Bolivia.

frío y por la esterilidad de la tierra, reportó que no existían granos, yerbas o frutos en el área. La única vegetación reportada que ha crecido en el área fueron algunos quishuar (árboles enanos) e ichu (una mata de yerbas tipo grass), no comestibles para los humanos y usada ocasionalmente como forraje para los camélidos. Ninguno de los más resistentes tubérculos o granos, como la quinua, creció en esta altura. La fauna nativa fue también muy limitada consistiendo en dos especies salvajes de camélidos, vicuñas y guanacos, así como venados y vizcachas (un mamífero de tamaño pequeño-mediano conocido como la liebre andina) (Jiménez de la Espada 1965
[1603]:373). De este modo, los residentes de Potosí y comunidades circundantes fueron obligados a importar la mayoría de sus productos alimenticios (ver Cobb 1949).

Las fuentes históricas sugieren que hacia el siglo XVI se había desarrollado un intercambio consistente en un flujo uniforme de plata proveniente de la capital a cambio de comestibles y otros productos de mercado (Acarete du Biscay 1968 [1698]) y que las ganancias económicas de este intercambio rivalizaron en importancia con Cerro Rico (Cobb 1949). Las rutas de intercambio comercial norte y sur de Potosí llevaron bienes legales e ilícitos 
desde y hacia la ciudad. Hacia fines del siglo XVI una ruta de transporte terrestre desde Potosí hasta el puerto de Arica (en el Chile actual) fue usada para el movimiento de plata. Arica está a sólo 1 grado de latitud norte de Potosí y a una distancia lineal de alrededor casi $500 \mathrm{~km}$ (300 millas); sin embargo, los viajeros terrestres usando el camino real tuvieron que atravesar una peligrosa y lenta ruta cruzando parte de la cordillera oriental, del altiplano boliviano y de la cordillera occidental de los Andes antes de descender hacia la costa del Pacífico. Hacia el sur de Potosí también se establecieron rutas de intercambio hacia Argentina y otros destinos sureños, particularmente para el transporte de productos alimenticios.

El mercado de Potosí está descrito repleto con suntuosos comestibles favoritos entre los habitantes más ricos (de Acosta 2002 [1590]:173; Cieza de León 1964 [1553]; Jiménez de la Espada 1965 [1603]:vol I 372-385). Los indígenas que trabajaban para los comerciantes españoles son mencionados como cargadores de fruta, granos, carne, yerbas y forraje para los animales desde los valles fértiles de Cochabamba, Petantora y Chuquisaca (La Plata colonial; Sucre moderno). Un recuento anual para el año 1603 indica que un estimado de 50.000 ovejas fueron criadas en las proximidades de la ciudad en elevaciones más bajas; además, 100.000 cabezas de llama llegaron a la ciudad y fueron consumidas. El consumo anual de ganado está reportado en 4.000 cabezas por año. Los residentes de la ciudad obtenían grandes cantidades de manteca de cerdo, tocino, jamones, lomos, lengua de cerdo y queso de las regiones de Tarija y Paria al sur de Potosí. Grandes cantidades de pescado salado llegaban desde Arica y otras localidades costeras. El pescado fresco llegaba desde los lagos de la sierra conservado en hielo. Los pescados no salados incluían suches (pez gato o bagre, probablemente Trichomycterus rivulatus) de la Laguna de Chucuito (Lago Titicaca) así como sábalos y dorados (peces de aguadulce) de los ríos cerca de Cochabamba y otras regiones. Gallinas, gallos, cabritos o perdices, y vizcachas (Lagidium peruanum) fueron disponibles a través de todo el año. La carne seca o charqui, presumiblemente carne de camélido, también fue común. Interesantemente, no existe en el trabajo de Jiménez de la Espada mención alguna sobre los cuyes indígenas, carne de venado o carne de camélido salvaje (p.ej., guanacos o vicuñas); sin embargo, los venados y camélidos salvajes son reportados a través de toda la región de La Plata al sur de Potosí (Acarete du Biscay 1968 [1698]).

Los animales y productos animales fueron usados también para una variedad de fines industriales (ver Bakewell 1988). Las llamas y/o mulas fueron necesarias para el transporte. Como los caballos eran costosos, así como un símbolo de riqueza y prestigio (Lockhart 1994), ellos fueron probablemente usados para el transporte exclusivo de los ricos. Los caballos y las mulas como fuerza motriz para los molinos fueron usados hacia la segunda mitad del siglo XVI, a pesar que el agua ya comenzaba a ser una fuerza motora más común. Inicialmente las llamas proporcionaron la grasa animal para las velas y la piel para las bolsas y otros objetos (p.ej., zapatos y ropa, escaleras de mano y ribetes). Eventualmente, los ranchos de ganado suplían estos productos. La grasa animal, tanto blanca como quemada, fue también usada como lubricante para la maquinaria de madera. Las haciendas que estaban destinadas principalmente a la producción de ganado doméstico y a proporcionar materia prima para la mina no prosperaron hasta la segunda mitad del siglo XVII (Bakewell 1988:142-143). Éstas estaban ubicadas tanto al norte como al sur del centro de la mina en las elevaciones más bajas y acogedoras para el ganado. Antes de la amplia aparición de las haciendas, los animales podrían haberse criado en ranchos o estancias que fueron parte de los propietarios de tierras controladas por los encomenderos españoles (Presta 1992). El registro zooarqueológico proporciona una medida empírica de estos informes históricos y nos permite examinar el surgimiento de los hábitos alimenticios ibero-andinos en la Bolivia colonial.

\section{Los Contextos Arqueológicos}

\section{Cruz Pampa}

El centro colonial de Porco, ubicado aproximadamente a $50 \mathrm{~km}$ (31 millas) al sudoeste de Potosí, fue una comunidad minera intensamente involucrada en la producción inicial de plata que comenzó en el siglo XVI. A pesar que varios sitios asociados con Porco han sido excavados, los restos de fauna mejor conservados proceden del sitio de Cruz Pampa ubicado a $300 \mathrm{~m}$ al sudeste de Porco. Van Buren y su equipo realizaron aquí excavaciones en varias estaciones de campo. Los depósitos de Cruz Pampa corresponden a material de basurales de las estructuras 
domésticas (Unidades 8 y 10 y Estructura 3). Una estructura contuvo un fogón de estilo indígena o $k$ 'oncha asociado al piso, sugiriendo la presencia de mujeres indígenas en el sitio. Los depósitos del sitio también proporcionaron un zapato de hombre de estilo europeo, una pipa, y algunas joyas sugiriendo que el sitio estaba asociado con un indígena masculino de estatus relativamente alto, posiblemente un yanacona o un español de bajo estatus. El sitio ha sido interpretado como una residencia habitada por individuos supervisores de la producción minera. Los depósitos datan aproximadamente de 1.6001.650 d.C. siendo contemporáneos con los restos de fauna procedentes de Tarapaya.

\section{Tarapaya}

La comunidad de Tarapaya está ubicada aproximadamente a $15 \mathrm{~km}$ ( 9 millas) al noroeste de Potosí. A una elevación de 3.300 msm (11.000 pies) la aldea está $700 \mathrm{~m}$ (2.200 pies) más abajo que Potosí. Como resultado de su baja elevación, Tarapaya es más cálida y su medio ambiente es menos tóxico que Potosí; por lo tanto las funciones respiratorias y del corazón son menos forzadas. Asimismo, Tarapaya es la ubicación de una serie de manantiales justo al norte de la aldea que sirvieron como baños terapéuticos tanto en el pasado como hoy en día. Los manantiales calientes, incluyendo un gran lago circular y una serie de pequeñas piscinas, fueron conocidos durante la época prehispánica aumentando su popularidad durante el control Inca de la región, como ha sido señalado por el historiador del siglo XVIII Arzáns de Orsúa y Vela (1965 [1787]:21 Volumen I, Libro 1); de igual manera, durante los siglos XVI y XVII fueron a menudo visitados por los residentes coloniales más ricos.

Durante el siglo XVII, próximo a los manantiales, existió un hostal comercial manejado por un individuo desconocido, así como una serie de casas pertenecientes a Francisco Gómez de la Rocha, un residente notable de Potosí y administrador del gobierno local, quien hizo su fortuna de la plata (Arzáns de Orsúa y Vela 1965 [1787] [Volumen II Libro IX]). Tanto el hostal como las residencias de campo fueron propiedades privadas.

Van Buren (1999:112-115) condujo mapeos, cateos y excavaciones arqueológicas en el sitio de los manantiales de Tarapaya. Los investigadores usaron los rasgos arquitectónicos en superficie para guiar las excavaciones en dos áreas del sitio. Los restos de fauna incluidos en este estudio proceden de los depósitos estratigráficos de basura adyacentes al gran lago circular (Unidades 1 y 2) y de los contextos asociados con el hostal y la residencia (Estructura 3, Recinto 3) así como de los depósitos estratigráficos de desechos a lo largo de la pared sur externa del edificio (Unidad 9). Aunque los visitantes del hostal pueden haber sido de diferentes procedencias étnicas (p.ej., mestizos de elite, criollos o peninsulares), todos fueron probablemente ricos. La preparación de la comida y el consumo de los alimentos podría haber sido en el hostal, ya que existían muy pocos servicios en el área de los manantiales. No logramos identificar áreas de preparación de alimentos en el sitio Tarapaya similares a las de fogones de cocina indígena excavados en Cruz Pampa. Por esa razón, no sabemos si las técnicas de cocina indígena, o europea, fueron utilizadas en Tarapaya. La cultura material y el registro histórico sugieren que los depósitos datan principalmente de la primera mitad del siglo XVII, aunque el rango de ocupación pudo haber sido mayor (Van Buren 1999:114).

\section{Metodología Zooarqueológica}

Todos los restos de fauna procedentes de Tarapaya y Cruz Pampa fueron recuperados con una malla de 1/4" (6,35 mm). Se combinaron analíticamente las muestras de cada sitio según la medida de su cantidad relativa. Para esto se realizó un análisis preliminar de los restos de fauna de Tarapaya y Cruz Pampa en Bolivia. La Unidad Nacional de Arqueología (UNAR) extendió el permiso para exportar el material con el fin de realizar estudios más detallados. La identificación de los restos de fauna se completó usando colecciones comparativas del Laboratorio de Arqueología Ambiental y de la Colección de Ornitología del Museo de Historia Natural de Florida, Universidad de Florida. Todos los restos zooarqueológicos fueron identificados en el nivel taxonómico más bajo posible. Las medidas de cantidad relativa que se emplearon son Número de Especímenes Identificados (NEI), estimados de Número Mínimo de Individuos (NMI) y peso del hueso.

También se calculó la frecuencia de las partes de esqueleto de mamíferos para determinar qué partes de la carcasa están representadas y estimar patrones de consumo. Las frecuencias relativas de las partes del esqueleto pueden ser usadas para estimar dos 
cuestiones relacionadas al uso de animales coloniales. Primero, el porcentaje de las partes del esqueleto puede ser usado para determinar si la cantidad de carne consumida representa la alta calidad de cortes de la carcasa. Segundo, las frecuencias de las partes del esqueleto proporcionan un indicador de la adquisición y descarnado de los animales.

Se tabularon las modificaciones de los huesos incluyendo descarnado, roído y quemado para estimar los efectos de los procesos tafonómicos naturales y culturales en la muestra. Las modificaciones en los huesos pueden ser también usadas para identificar patrones culturales de procesamiento, preparación $\mathrm{y}$ desecho de animales.

\section{Resultados}

\section{Cruz Pampa}

La muestra de Cruz Pampa en Porco consistió en 4.465 especímenes representando un mínimo de 45 individuos (Tabla 1). La fauna representada consiste en ocho especies de mamíferos y tres especies de aves. La muestra está dominada por los camélidos (Lama spp. y Camelidae), tanto en sus variedades grande y pequeña, siendo los camélidos grandes los más comunes. En términos de NEI, los camélidos constituyen más del $90 \%$ de la muestra (Figura 2). Los restos de los mamíferos euroasiáticos, incluyendo ganado vacuno (Bos taurus), caprinos (Caprinae), y caballos o burros (Equus sp. and Equus cf. caballus), están representados pero en pequeñas cantidades. También están presentes los restos de perro (Canis familiaris), cuy (Cavia porcellus) y roedores no identificados (Rodentia). Los roedores y los perros probablemente no representan desechos de comida.

Las especies de aves están representadas por el cóndor andino (Vultur gryphus), un tipo de chotacabras (Caprimulgidae), y pollos (Gallus gallus). Ninguno de estos es abundante. Ninguno de los restos de pollos pertenece a gallinas ponedoras. También están presentes restos de aves no identificadas de tamaño mediano.

No son comunes el descarnado y modificaciones del hueso (Tabla 2). Los tajos son más comunes que los cortes de cuchillos. Algunos huesos exhiben pulido sugiriendo su uso como herramientas. Los huesos quemados, de color negro o blanco y negro, son más comunes que los parcialmente negros (quemado resultante de cocina). Un pequeño número de especímenes de

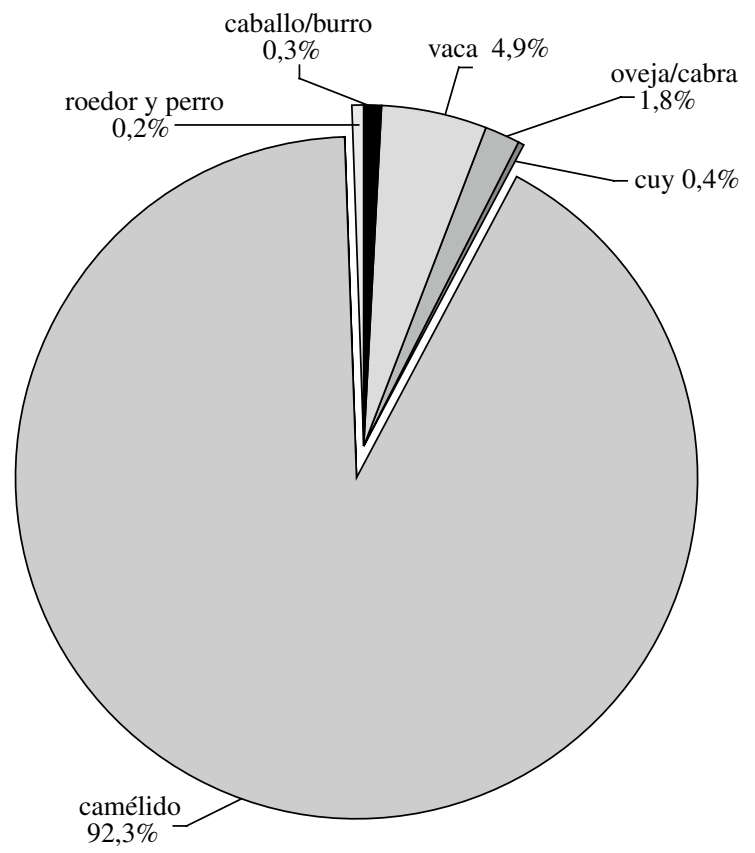

Figura 2. Porcentajes de NEI (Número de Especies Identificadas) para mamíferos, Cruz Pampa. Percent NISP (Number of Identified Specimens) of mammals, Cruz Pampa. 
Tabla 1. Restos de fauna y presencia relativa, Cruz Pampa. Identified faunal remains and relative abundance, Cruz Pampa.

\begin{tabular}{|c|c|c|c|c|c|c|c|}
\hline Taxa & Nombre común & NEI & $\%$ & NMI & $\%$ & Peso $(\mathrm{g})$ & $\%$ \\
\hline Rodentia nn & roedor no identificado & 1 & 0,0 & 1 & 2,2 & 0,5 & 0,0 \\
\hline Cavia porcellus & cuy & 4 & 0,1 & 1 & 2,2 & 6,2 & 0,0 \\
\hline Canis familiaris & perro & 1 & 0,0 & 1 & 2,2 & 23,0 & 0,1 \\
\hline Equus sp. & caballo/burro/mula & 1 & 0,0 & 1 & 2,2 & 33,2 & 0,1 \\
\hline Equus cf. caballus & cf. caballo & 1 & 0,0 & 0 & 0,0 & 67,5 & 0,3 \\
\hline Equidae & caballo/burro/mula & 1 & 0,0 & 1 & 2,2 & 29,3 & 0,1 \\
\hline Bos taurus & vaca & 44 & 1,0 & 6 & 13,3 & $1.463,3$ & 5,7 \\
\hline Caprinae & oveja/cabra & 14 & 0,3 & 4 & 8,9 & 173,0 & 0,7 \\
\hline cf. Caprinae & cf. oveja/cabra & 2 & 0,0 & 0 & 0,0 & 18,0 & 0,1 \\
\hline Bovidae/Camelidae & vaca/camélido & 13 & 0,3 & 0 & 0,0 & 45,9 & 0,2 \\
\hline Bovidae/Equidae & vaca/caballo & 2 & 0,0 & 0 & 0,0 & 37,1 & 0,1 \\
\hline Lama spp. & llama/alpaca & 731 & 16,4 & 15 & 33,3 & $11.388,0$ & 44,0 \\
\hline Lama sp. (pequeño) & camélido pequeño & 10 & 0,2 & 4 & 8,9 & con Lama spp. & \\
\hline Lama sp. (grande) & camélido grande & 82 & 1,8 & 6 & 13,3 & con Lama spp. & \\
\hline cf. Lama sp. & cf. camélido & 1 & 0,0 & 0 & 0,0 & 1,5 & 0,0 \\
\hline Mamífero grande nn & mamífero grande no identificado & 3.501 & 78,4 & 0 & 0,0 & $12.557,2$ & 48,5 \\
\hline Mamífero nn & mamífero pequeño no identificado & 47 & 1,1 & 0 & 0,0 & 12 & 0,0 \\
\hline Total Mammalia & total mamíferos & 4456 & 99,8 & 40 & 88,9 & $25.856,3$ & 99,9 \\
\hline Vultur gryphus & cóndor andino & 1 & 0,0 & 1 & 2,2 & 13,3 & 0,1 \\
\hline Caprimulgidae & chotacabras & 1 & 0,0 & 1 & 2,2 & 0,1 & 0,0 \\
\hline Gallus gallus & gallo/pollo & 4 & 0,1 & 2 & 4,4 & 6,2 & 0,0 \\
\hline Aves nn-med & pájaros no identificados & 2 & 0,0 & 0 & 0,0 & 2,6 & 0,0 \\
\hline Aves nn & pájaros no identificados & 1 & 0,0 & 1 & 2,2 & 0,4 & 0,0 \\
\hline Total aves & total pájaros & 9 & 0,2 & 5 & 11,1 & 22,6 & 0,1 \\
\hline Vertebrados nn & vertebrados no identificados & $\mathrm{n} / \mathrm{c}$ & 0,0 & 0 & 0,0 & 3,9 & 0,0 \\
\hline Muestra Total & & 4.465 & 100,0 & 45 & 100,0 & $25.882,8$ & 100,0 \\
\hline
\end{tabular}

Tabla 2. Sumario de huesos modificados y quemados, Cruz Pampa. Summary of bone modifications and burning, Cruz Pampa.

\begin{tabular}{|c|c|c|c|c|c|c|}
\hline Modificados & Cortes & Tajos & Tajos y cortes & $\begin{array}{l}\text { Roídos por } \\
\text { carnívoros }\end{array}$ & $\begin{array}{l}\text { Pulidos o } \\
\text { trabajados }\end{array}$ & Total \\
\hline \multicolumn{7}{|l|}{ Taxón } \\
\hline Bos taurus & 1 & 6 & 1 & 0 & 0 & 8 \\
\hline Bovidae/Camelidae & 0 & 1 & 0 & 0 & 0 & 1 \\
\hline Caprinae & 0 & 3 & 0 & 0 & 0 & 3 \\
\hline Cavia porcellus & 1 & 0 & 0 & 0 & 0 & 1 \\
\hline Lama spp. & 81 & 251 & 31 & 19 & 15 & 397 \\
\hline Canis familiaris & 1 & 0 & 1 & 0 & 0 & 2 \\
\hline Gallus gallus & 1 & 0 & 0 & 0 & 0 & 1 \\
\hline Total & 83 & 261 & 33 & 19 & 15 & 411 \\
\hline Porcentaje del total & 20,2 & 63,5 & 8,0 & 4,6 & 3,6 & 100,0 \\
\hline Quemados & pne & ne & ne/bl & bl & & \\
\hline Unidad 8 & 121 & 423 & 222 & 74 & & \\
\hline Unidad 10 & 7 & 144 & 41 & 0 & & \\
\hline Estructura 3 & 0 & 13 & 78 & 2 & & \\
\hline Total & 128 & 580 & 341 & 76 & & \\
\hline
\end{tabular}


Tabla 3. Representación del esqueleto de mamíferos, Cruz Pampa. Representation of mammalian skeletal elements, Cruz Pampa.

\begin{tabular}{lrr}
\hline Lama spp. & & \\
\hline Partes & \multicolumn{1}{c}{$\#$} & $\%$ \\
\hline Craneal & 98 & 11,9 \\
Axial & 199 & 24,2 \\
Extremidades delanteras & 134 & 16,3 \\
Extremidades traseras & 71 & 8,6 \\
Patas delanteras & 22 & 2,7 \\
Patas traseras & 133 & 16,2 \\
Patas & 166 & 20,2 \\
Total & 823 & 100,0 \\
& & \\
\hline
\end{tabular}

\begin{tabular}{lcr}
\hline Bos taurus & & \\
\hline Partes & $\#$ & $\%$ \\
\hline Craneal & 8 & 17,0 \\
Axial & 1 & 2,1 \\
Extremidades delanteras & 2 & 4,3 \\
Extremidades traseras & 3 & 6,4 \\
Patas delanteras & 9 & 19,1 \\
Patas traseras & 4 & 8,5 \\
Patas & 20 & 42,6 \\
Total & 47 & 100,0 \\
& & \\
\hline
\end{tabular}

\begin{tabular}{lrr}
\hline Caprinae & & \\
\hline Partes & $\#$ & $\%$ \\
\hline Craneal & 0 & 0,0 \\
Axial & 0 & 0,0 \\
Extremidades delanteras & 2 & 14,3 \\
Extremidades traseras & 0 & 0,0 \\
Patas delanteras & 0 & 0,0 \\
Patas traseras & 10 & 71,4 \\
Patas & 2 & 14,3 \\
Total & 14 & 100,0 \\
\hline
\end{tabular}

mamíferos $(\mathrm{n}=19)$ presenta roídos de carnívoros. Patologías del esqueleto están presentes sólo en falanges de bóvidos sugiriendo el uso de estos animales para tracción u otros fines utilitarios (deFrance 2010).

La representación de diferentes elementos del esqueleto de mamíferos comestibles se muestra en la Tabla 3 y Figura 3. Estos valores son los porcentajes del número de elementos, más que porcentajes del número mínimo de partes del esqueleto; de esta manera, la cantidad relativa de partes que son representadas por muchos elementos o que son representadas por elementos altamente diagnósticos (p.ej., elementos axiales y patas) no son sobrerrepresentados en la muestra. Los camélidos y el ganado están representados por el rango mayor de elementos del esqueleto incluyendo todas las partes del esqueleto. La pequeña muestra de especímenes caprinos procede sólo de las extremidades delanteras, patas traseras y patas. Mientras que la representación de los especímenes de camélidos y ganado vacuno indica que los animales completos estuvieron presentes y fueron descarnados cercanamente. Todas las partes del esqueleto de vacunos están presentes, pero más del $87 \%$ de los especímenes proceden de cortes de baja utilidad de la cabeza y las patas. Interesantemente, el ganado puede haber servido para un propósito doble como animales utilitarios y para alimento, ya que la muestra incluye tanto partes del esqueleto con descarnado como especímenes (falanges) con patologías posiblemente relacionadas con trabajo.

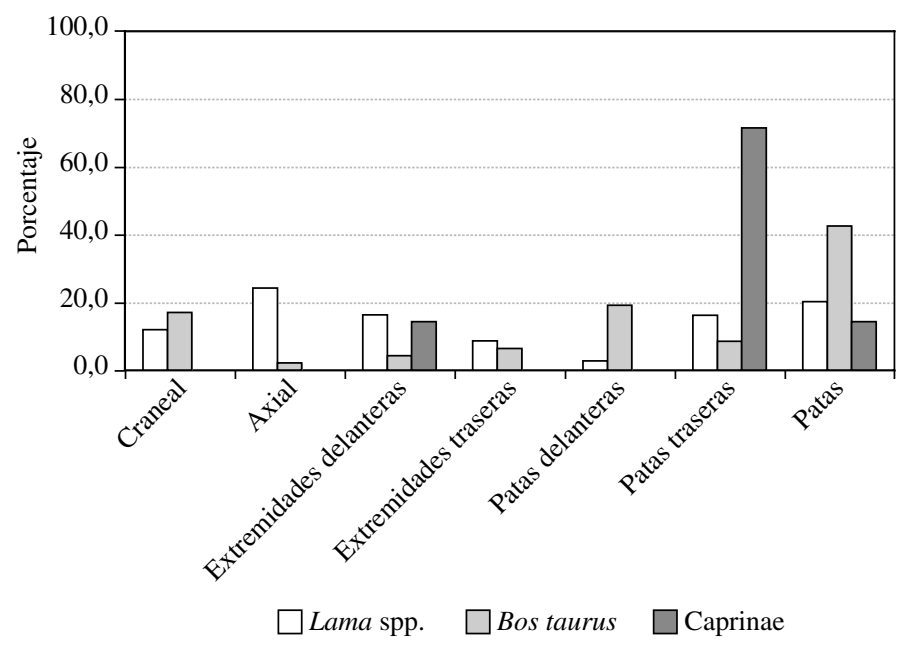

Figura 3. Representación de los elementos de camélidos, Cruz Pampa.

Representation of camelid elements, Cruz Pampa. 
Tabla 4. Restos de fauna y presencia relativa, Tarapaya.

Identified faunal remains and relative abundance, Tarapaya.

\begin{tabular}{|c|c|c|c|c|c|c|c|}
\hline Taxa & Nombre común & NEI & $\%$ & NMI & $\%$ & Peso (g) & $\%$ \\
\hline Cavia porcellus & cuy & 18 & 0,1 & 3 & 1,9 & 10,2 & 0,0 \\
\hline Chinchilla sp. & chinchilla & 1 & 0,0 & 1 & 0,6 & 0,6 & 0,0 \\
\hline Lagidium peruanum & vizcacha & 5 & 0,0 & 2 & 1,3 & 6,1 & 0,0 \\
\hline Felis catus & gato doméstico & 1 & 0,0 & 1 & 0,6 & 1,7 & 0,0 \\
\hline Equus sp. & caballo, mula, burro & 8 & 0,1 & 2 & 1,3 & 457,1 & 1,6 \\
\hline Sus scrofa & chancho & 72 & 0,6 & 3 & 1,9 & 515,2 & 1,9 \\
\hline Bos taurus & vaca & 127 & 1,0 & 3 & 1,9 & $2.741,2$ & 9,9 \\
\hline Caprinae & oveja/cabra & 1.356 & 10,7 & 33 & 21,2 & $7.701,4$ & 27,7 \\
\hline Ovis aries & oveja & 2 & 0,0 & 1 & 0,6 & 25,9 & 0,1 \\
\hline Lama spp. & 1lama, alpaca & 28 & 0,2 & 2 & 1,3 & 389,4 & 1,4 \\
\hline Mammalia nn & mamífero no identificado & 7.805 & 61,6 & 0 & 0,0 & $14.306,4$ & 51,5 \\
\hline Mamífero pequeño nn & $\begin{array}{l}\text { mamífero pequeño no } \\
\text { identificado }\end{array}$ & 6 & 0,0 & 0 & 0,0 & 1,4 & 0,0 \\
\hline Total Mamíferos & & 9.429 & 74,5 & 61 & 39,1 & $26.156,6$ & 94,2 \\
\hline Gallus gallus & gallina & 673 & 5,3 & 37 & 23,7 & 564,9 & 2,0 \\
\hline Nothura maculosa & tinamú manchado & 1 & 0,0 & 1 & 0,6 & 0,1 & 0,0 \\
\hline Ortalis sp. & chachalaca & 9 & 0,1 & 8 & 5,1 & 3,9 & 0,0 \\
\hline Tinamidae & tinamús & 20 & 0,2 & 3 & 1,9 & 9,8 & 0,0 \\
\hline Galliformes & aves gallináceas & 173 & 1,4 & 0 & 0,0 & 77,0 & 0,3 \\
\hline Columbidae & palomas & 2 & 0,0 & 1 & 0,6 & 0,3 & 0,0 \\
\hline Columbidae cf. Metriopelia sp. & paloma & 1 & 0,0 & 1 & 0,6 & 0,1 & 0,0 \\
\hline Aves nn & pájaros no identificados & 1.221 & 9,6 & 0 & 0,0 & 486,4 & 1,8 \\
\hline Total Aves & & 2.100 & 16,6 & 52 & 33,3 & $1.142,5$ & 4,1 \\
\hline Anuro & rana & 2 & 0,0 & 1 & 0,6 & 0,2 & 0,0 \\
\hline Total Anfibios & & 2 & 0,0 & 1 & 0,6 & 0,2 & 0,0 \\
\hline Carcharhinidae & tiburones del requiem & 1 & 0,0 & 1 & 0,6 & 0,2 & 0,0 \\
\hline Lamniformes & tiburones & 2 & 0,0 & 1 & 0,6 & 0,6 & 0,0 \\
\hline Chondrichthyes nn & $\begin{array}{l}\text { pescados cartilaginosos } \\
\text { no identificados }\end{array}$ & 2 & 0,0 & 0 & 0,0 & 0,4 & 0,0 \\
\hline Total Chondrichthyes & & 5 & 0,0 & 2 & 1,3 & 1,2 & 0,0 \\
\hline Trichomycterus sp. & bagre/suche & 4 & 0,0 & 2 & 1,3 & 0,7 & 0,0 \\
\hline Siluriformes & siluros & 11 & 0,1 & 3 & 1,9 & 4,9 & 0,0 \\
\hline Genypterus sp. & anguila & 201 & 1,6 & 13 & 4,5 & 140,2 & 0,5 \\
\hline Orestias sp. & corvinilla/ispi & 8 & 0,1 & 4 & 5,1 & 0,7 & 0,0 \\
\hline Bodianus sp. & viejo & 2 & 0,0 & 1 & 0,6 & 2,2 & 0,0 \\
\hline Prochilodus sp. & sábalo & 142 & 1,1 & 13 & 8,3 & 67,2 & 0,2 \\
\hline Osteichthyes nn & $\begin{array}{l}\text { huesos de pescados no } \\
\text { identificados }\end{array}$ & 759 & 6,0 & 3 & 0,0 & 236,4 & 0,9 \\
\hline Total Osteichthyes & & 1.127 & 8,9 & 40 & 25,0 & 452,3 & 1,6 \\
\hline Vertebrados nn & $\begin{array}{l}\text { vertebrados no } \\
\text { identificados }\end{array}$ & $\mathrm{n} / \mathrm{c}$ & & 0 & 0,0 & 55,6 & 0,2 \\
\hline Total Vertebrados & & 12.663 & 100,0 & 155 & 99,4 & $27.808,4$ & 100,2 \\
\hline Bivalvos marinos nn & & 1 & 0,0 & 1 & 0,6 & 0,9 & 0,0 \\
\hline Muestra Total & & 12.664 & 100,0 & 156 & 100,0 & $27.753,7$ & 100,0 \\
\hline
\end{tabular}




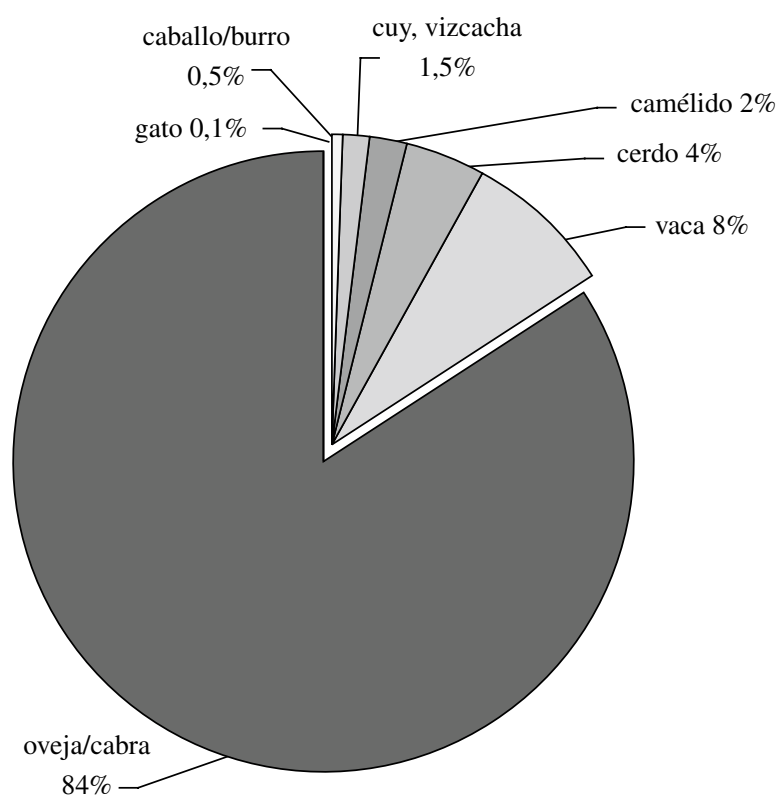

Figura 4. Porcentajes de NEI (Número de Especies Identificadas) para mamíferos, Tarapaya. Percent NISP (Number of Identified Specimens) of mammals, Tarapaya.

\section{Tarapaya}

La muestra de Tarapaya consiste de 12.664 especímenes (Tabla 4). Sólo uno de éstos es un espécimen invertebrado: un fragmento de bivalvo marino. Un mínimo de 157 individuos está representado en la muestra.

Los mamíferos están representados por lo menos por nueve especies. Los mamíferos domésticos de origen euroasiático dominan la muestra. De lejos, la especie doméstica más abundante en todas las medidas de presencia relativa $(\mathrm{n}=1.356 ; \mathrm{NMI}=33)$ son los caprinos (Caprinae, ovejas o cabras) (Figura 4). Presumiblemente, están representadas las ovejas y cabras; sin embargo, es muy difícil distinguir a través de la osteología las diferencias entre ambas. Sólo dos elementos pudieron ser positivamente identificados como una oveja (Ovis aries). Descendiendo en abundancia están el ganado vacuno (Bos taurus), cerdos (Sus scrofa) y equinos (caballos, burros o mulas). Sólo está presente un elemento de gato doméstico (Felis catus). Los mamíferos nativos están representados por llamas o alpacas (Lama spp.), cuyes (Cavia porcellus), vizcacha (Lagidium peruanum), y un elemento de una chinchilla (Chinchilla sp.). El gato doméstico y la chinchilla son mamíferos que probablemente no representen desechos de comida. De otro lado, el pequeño número de restos de equinos podría representar restos de comida o desechos no alimenticios que fueron descartados en el área.

Las aves están representadas por lo menos por cuatro especies. La muestra de restos de aves es grande ( $\mathrm{n}=2.100 ; \mathrm{NMI}=52)$. Los abundantes restos de pollos (Gallus gallus) dominan la muestra. De las aves, los pollos y los restos inmaduros no identificados (orden de los Galliformes) contabilizan el $71 \%$ del NMI y el $40 \%$ de los fragmentos. Por lo menos doce gallinas ponedoras están presentes indicadas por la identificación del hueso medular, un depósito esponjoso que se acumula en los huesos largos de aves hembras en condición de ponedoras. Otras aves presentes en la muestra son las especies nativas del tinamú manchado (Nothura maculosa), chachalaca (Ortalis sp.) y palomas (Columbidae cf. Metriopelia sp.). También se encontraron restos de huevos en algunos contextos, pero no fueron cuantificados.

Considerando la ubicación de altura, los restos de pescados son muy diversos. La muestra contiene restos de peces cartilaginosos y óseos. Por lo menos están presentes dos tipos de tiburones (Carcharhinidae y Lamniformes). Los tiburones pueden emigrar grandes distancias tierra adentro a través de los ríos 
Tabla 5. Sumario de huesos modificados y quemados, Tarapaya. Summary of bone modifications and burning, Tarapaya.

\begin{tabular}{|c|c|c|c|c|c|c|c|}
\hline Modificados & Cortes & Tajos & $\begin{array}{l}\text { Tajos y } \\
\text { cortes }\end{array}$ & $\begin{array}{l}\text { Roídos por } \\
\text { carnívoros }\end{array}$ & $\begin{array}{l}\text { Roídos por } \\
\text { roedores }\end{array}$ & $\begin{array}{l}\text { Pulidos o } \\
\text { trabajados }\end{array}$ & Total \\
\hline \multicolumn{8}{|l|}{ Taxón } \\
\hline Sus scrofa & 5 & 1 & 0 & 1 & 0 & 0 & 7 \\
\hline Bos taurus & 20 & 6 & 5 & 4 & 0 & 0 & 35 \\
\hline Caprinae & 101 & 49 & 43 & 34 & 0 & 4 & 231 \\
\hline Lama sp. & 2 & 0 & 0 & 1 & 0 & 0 & 3 \\
\hline Mamífero no identificado & 616 & 629 & 0 & 88 & 2 & 0 & 1.335 \\
\hline Mamífero pequeño no identificado & 1 & 0 & 0 & 0 & 0 & 0 & 1 \\
\hline Mamífero grande no identificado & 0 & 0 & 0 & 2 & 0 & 0 & 2 \\
\hline Ortalis sp. & 1 & 0 & 0 & 0 & 0 & 0 & 1 \\
\hline Tinamidae & 1 & 0 & 0 & 0 & 0 & 0 & 1 \\
\hline Galliformes & 13 & 3 & 12 & 0 & 0 & 0 & 28 \\
\hline Gallus gallus & 69 & 2 & 24 & 0 & 0 & 0 & 95 \\
\hline Aves no identificadas & 29 & 0 & 0 & 2 & 0 & 0 & 31 \\
\hline Taxón & & & & & & & 0 \\
\hline Genypterus sp. & 2 & 0 & 0 & 0 & 0 & 0 & 2 \\
\hline Osteichthyes no identificados & 4 & 0 & 0 & 0 & 0 & 0 & 4 \\
\hline Total & 864 & 690 & 84 & 132 & 2 & 4 & 1.776 \\
\hline Porcentaje de total & 48,6 & 38,9 & 4,7 & 7,4 & 0,1 & 0,2 & 100,0 \\
\hline $\begin{array}{l}\text { Quemados } \\
\text { Taxón }\end{array}$ & pne & ne & ne/bl & bl & & & \\
\hline Gallus gallus & & & & 1 & & & \\
\hline Galliformes & & 3 & & & & & \\
\hline Aves no identificadas & & & 1 & & & & \\
\hline Caprinae & 2 & 3 & 1 & 3 & & & \\
\hline Mamífero no identificado & 35 & 82 & 4 & 22 & & & \\
\hline Total & 37 & 88 & 6 & 26 & & & \\
\hline
\end{tabular}

pne $=$ parcialmente negro, ne $=$ negro, ne/bl $=$ negro $/$ blanco, $\mathrm{bl}=$ blanco .

de Sudamérica y han sido capturados a distancias de la costa, además el Océano Pacífico es el hogar de numerosas especies. Los peces óseos incluyeron especies marinas, de aguas dulces y lacustres de altura. Las anguilas (Genypterus sp.) son los restos de peces marinos más comunes; por lo menos trece individuos están presentes. En segundo lugar de los peces marinos está el viejo (Bodianus sp.). La anguila y el viejo pueden haber sido obtenidos de la Costa Pacífica. Aparentemente, los peces marinos llegaron completos desde la costa ya que están presentes numerosos elementos craneales. Peces lacustres de altura incluyen los restos de corvinilla (Orestias spp.) y bagre (Trichomycterus spp.). Ambos son abundantes en la actualidad en el Lago Titicaca y en el Lago Poopó. También están presentes por lo menos dos tipos de peces de agua dulce: el sábalo y el bagre. El sábalo (Prochilodus spp.) es una especie que se alimenta del fondo ribereño y que actualmente habita el río Pilcomayo al sur de Potosí, así como otros numerosos ríos de Sudamérica; esta especie está representada en trece individuos. Además del sábalo también están presentes por lo menos tres bagres no-lacustres (Siluriformes).

Otros restos incluyen dos elementos de una pequeña rana o sapo (Anura) y un fragmento de un bivalvo marino. El fragmento de bivalvo podría representar desecho de comida, mientras que la rana/ sapo es interpretada como una especie comensal que no fue desecho de comida.

Las modificaciones de hueso presentes incluyen evidencia de descarnado, roído de animales y hueso trabajado o pulido (Tabla 5). La evidencia de descarnado se encuentra en los restos de mamíferos, aves y peces e incluye cortes y tajos. Los huesos que presentan cortes con cuchillo asociados con un 
Tabla 6. Representación del esqueleto de mamíferos, Tarapaya.

Representation of mammalian skeletal elements, Tarapaya.

\begin{tabular}{|c|c|c|}
\hline Lama spp. & $\#$ & $\%$ \\
\hline Craneal & 1 & 3,6 \\
\hline Axial & 8 & 28,6 \\
\hline Extremidades delanteras & 2 & 7,1 \\
\hline Patas delanteras & 2 & 7,1 \\
\hline Extremidades traseras & 1 & 3,6 \\
\hline Patas traseras & 2 & 7,1 \\
\hline Patas & 12 & 42,9 \\
\hline Total & 28 & 100,0 \\
\hline Bos taurus & $\#$ & $\%$ \\
\hline Craneal & 15 & 11,8 \\
\hline Axial & 47 & 37,0 \\
\hline Extremidades delanteras & 8 & 6,3 \\
\hline Patas delanteras & 7 & 5,5 \\
\hline Extremidades traseras & 7 & 5,5 \\
\hline Patas traseras & 6 & 4,7 \\
\hline Patas & 37 & 29,1 \\
\hline Total & 127 & 100,0 \\
\hline Sus scrofa & $\#$ & $\%$ \\
\hline Craneal & 16 & 22,2 \\
\hline Axial & 6 & 8,3 \\
\hline Extremidades delanteras & 4 & 5,6 \\
\hline Patas delanteras & 12 & 16,7 \\
\hline Extremidades traseras & 4 & 5,6 \\
\hline Patas traseras & 6 & 8,3 \\
\hline Patas & 24 & 33,3 \\
\hline Total & 72 & 100,0 \\
\hline Caprinae & $\#$ & $\%$ \\
\hline Craneal & 132 & 9,7 \\
\hline Axial & 779 & 57,4 \\
\hline Extremidades delanteras & 204 & 15,0 \\
\hline Patas delanteras & 20 & 1,5 \\
\hline Extremidades traseras & 140 & 10,3 \\
\hline Patas traseras & 41 & 3,0 \\
\hline Patas & 40 & 2,9 \\
\hline Total & 1.356 & 100,0 \\
\hline
\end{tabular}

procesamiento secundario y/o consumo son el tipo de modificación más común seguido por los tajos. Algunos elementos contienen tanto tajos como cortes pequeños. De los elementos de esqueleto modificados, más del $70 \%$ contiene evidencia de roídos por carnívoros. Un gato doméstico está representado en los restos de fauna; sin embargo, no se han encontrado restos de perros o individuos de tamaño grande. Sólo dos elementos presentan evidencias de roído por roedores. Cuatro especímenes de caprinos (podiales o epífisis) están trabajados y pulidos. Aunque la función de estos objetos es desconocida, ellos aparentan cubos inacabados.

Todas las partes del esqueleto están presentes en la muestra para cada mamífero de mayor valor alimenticio (camélidos, ganado, cerdos y caprinos) (Tabla 6; Figura 5). Las dos categorías más abundantes son la parte axial consistente en las vértebras y costillas y la parte de las patas consistentes de los indistinguibles metapodios, podiales y falanges. Un gran número de elementos representan ambas partes del esqueleto. En el caso de las patas, los elementos individuales son fácilmente identificables. Para la parte axial, las costillas y las vértebras están comúnmente descarnadas en un número de pequeños, pero todavía identificables, cortes de carne. Así como en Cruz Pampa, las cantidades de los especímenes de la muestra no son usados para estimar el número mínimo de partes del esqueleto. El rango de elementos sugiere que los animales completos fueron descarnados en las proximidades cercanas al hostal y a la residencia en Tarapaya. El patrón de descarte incluye una gran cantidad de huesos con cortes en el cuerpo medio y en las partes carnosas de las extremidades delanteras y traseras, así como el descarte de los elementos de las patas con bajo contenido de carne.

\section{Discusión}

Los contrastes entre las muestras de los dos sitios son sorprendentes. La muestra de Cruz Pampa está comprendida principalmente de fauna nativa disponible localmente. En ésta se observa poca variedad en las partes de carne consumidas. Existen algunos animales euroasiáticos, pero parece que ellos contribuyeron de una forma mínima en la dieta. Algunos caballos o burros son parte de la muestra, pero ellos podrían haber sido rápidamente adoptados como transporte y como bestias de carga. En el caso del ganado vacuno, éste podría haber sido consumido después que su utilidad para otros fines haya sido explotada. No hay aves o peces importados en la muestra de Cruz Pampa. A pesar que los residentes (individuos y familias) y trabajadores en Cruz Pampa, que son los responsables de la formación de los depósitos de basura, podrían haber estado en una posición administrativa relacionada a la minería y adquisición de algunos bienes materiales de origen europeo, no hay un indicativo de que ellos consumieran carne que pudiera ser considerada 


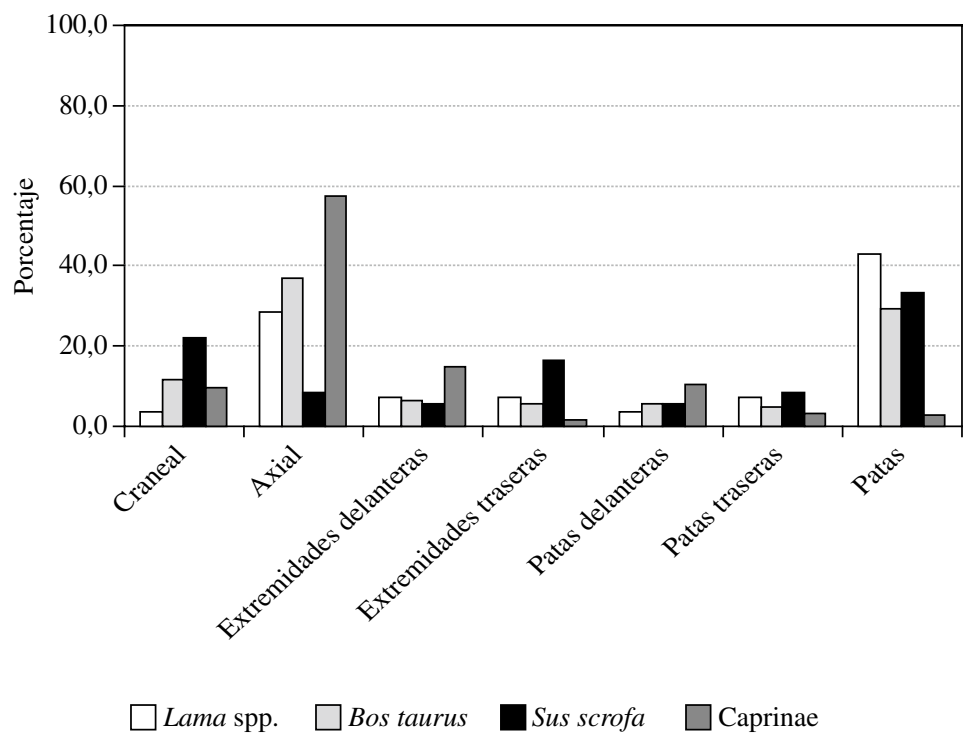

Figura 5. Representación de los elementos de camélidos, Tarapaya. Representation of camelid elements, Tarapaya.

española o ibérica, a excepción de alguna carne de cordero o cabra. También es obvia la ausencia de los medios o del deseo de obtener peces o aves de corral importados. Es difícil determinar si esto refleja la preferencia personal o la falta de acceso en los mercados y/o en Porco a fuentes de carne exótica. Los propietarios ricos de las minas de Porco y algunos españoles podrían haber residido en Porco por lo menos parte del tiempo, pero también muchos podrían haber tenido sus residencias en otras ciudades.

Porco se encuentra aislada y es muy inhóspita. Debido que está ubicada en una elevación más alta que Potosí o Tarapaya, es posible que los animales experimentaran un gran estrés hipóxico y no pudieran ser criados cerca de Porco hacia comienzos del siglo XVII. Sin embargo, los bienes y servicios relacionados a la minería pudieron fluir dentro de Porco con regularidad; de esta manera, los productos alimenticios también habrían sido transportados en caso de existir el deseo por tales productos. A pesar que haya existido la infraestructura y medios para la importación de alimentos no locales, existen pocos indicativos empíricos que las fuentes de alimentos no locales hayan comenzado a ser componentes más importantes en la dieta. El mayor acceso a animales de origen local junto con una preferencia étnica o personal por las fuentes de carne local podría estar sustentado por la predominancia de la fauna local en la muestra de Cruz Pampa. El matrimonio inter-étnico de un español o un indígena de cierto estatus con una mujer indígena también podría haber resultado en la preferencia y selección de animales comestibles nativos. Una mujer indígena con responsabilidades en la preparación de alimentos podría haber seleccionado fuentes de carne local sobre nuevos artículos comestibles. En el San Agustín español colonial, el matrimonio entre un español y una mujer indígena resultó en un patrón similar de uso de fauna local, probablemente como un resultado de la familiaridad femenina con los animales comestibles locales y sus métodos de preparación de alimentos (Deagan 1974).

En contraste con el uso de animales en Cruz Pampa, los habitantes de Tarapaya consumieron principalmente fauna introducida, especialmente caprinos y pollos. Si las fuentes históricas son correctas y unos pocos animales introducidos fueron criados cerca de Potosí, los habitantes de Tarapaya fueron capaces de adquirir fuentes de carne, incluyendo pescado fresco de hábitats lacustres, ribereños y marinos. Así como la plata dejó la ciudad, la carne y posiblemente los animales vivos fueron transportados para los residentes. El flujo de comestibles a la región no fue interrumpido por la elevación o la distancia. El gran número de gallinas en la muestra Tarapaya sugiere que sus habitantes tuvieron acceso a huevos frescos. 
En cuanto a la variedad y cantidad de fuentes de carne euroasiática en Tarapaya, la dieta fue más de carácter ibérico que en otras áreas de asentamientos españoles (p.ej., Perú, Caribe y la Florida española) (deFrance 1993, 1996; Reitz 1986, 1992; Reitz y McEwan 1995). Los habitantes también consumieron pequeñas cantidades de carne de alpaca, cuy, cerdo y vizcacha. Los residentes podrían haber experimentado con alimentos locales o la composición étnica de la gente asociada con Tarapaya podría haber incluido sirvientes indígenas o residentes que prefirieron estos tipos de fuentes de carne. Presumiblemente, las comidas hechas con carne de animales de Eurasia fueron preparadas por las mujeres de bajo estatus de ascendencia española o mujeres indígenas que habían sido instruidas en los métodos de la cocina ibérica. En contraste con Cruz Pampa y algunas otras zonas de la colonización española, no hay indicios de que las mujeres indígenas ejercieran un control significativo sobre el menú o los métodos de preparación.

La habilidad para adquirir carne asociada fuertemente con patrones de consumo españoles (p.ej., cordero, vaca y pollo) confirma el estatus probable de los individuos asociados con los depósitos de Tarapaya, así como el éxito de las rutas comerciales para el movimiento de productos alimenticios. Como ha sido discutido previamente (deFrance 2003; Van Buren 1999), la cultura material de Tarapaya no incluye muchos objetos de manufactura europea, posiblemente como un resultado del asentamiento rural; sin embargo, la dieta incluye abundancia de fuentes de carne euroasiática. Es evidente que los animales euroasiáticos se desarrollaron en algunas regiones de la Sierra peruana y que las rutas comerciales pudieron haberlos transportado exitosamente hasta Tarapaya. En ausencia de material cultural europeo, la dieta podría haber servido para distinguir a la población de origen español de la de origen indígena.

En el caso de Cruz Pampa, los individuos pudieron haber logrado un nivel de estatus administrativo en la economía minera, pero mantuvieron una dieta caracterizada por animales comestibles locales. En Tarapaya, la porción de carne en la dieta incluyó una gran variedad y gran cantidad de productos alimenticios importados. A pesar que se consumió un poco de carne nativa, es evidente una preferencia por los animales no locales. No obstante, las diferencias en elevación y condiciones medioambientales en los dos sitios, el determinante más fuerte en el uso de los animales parece estar relacionado con el estatus socioeconómico en combinación con las preferencias de la dieta.

El uso no relacionado con la dieta de los animales en ambos sitios es más difícil para identificar e interpretar. Existen restos de animales que son más comúnmente utilitarios (p.ej., caballos, burros) y existen restos de animales que sirven para propósitos duales como animales de trabajo y animales alimenticios (p.ej., ganado vacuno, camélidos). En Cruz Pampa, los animales de trabajo de origen euroasiático pueden haber sido usados para varias tareas. A pesar que algunos animales utilitarios están presentes y algunos restos exhiben patologías relacionadas con el trabajo, la poca frecuencia de animales de trabajo euroasiáticos sugiere que estos animales no fueron preferidos a los camélidos o que fueron usados pero que sus restos fueron descartados en otro sitio. Se necesitan muestras adicionales de fauna de otros sitios cerca de Porco así como medidas de los restos de fauna para determinar el tamaño de animales, a fin de poder determinar el posible rol utilitario de los animales euroasiáticos.

\section{Conclusiones}

Las muestras de fauna de dos sitios coloniales cerca de Potosí proporcionan observaciones empíricas acerca del uso de los animales durante la primera mitad del siglo XVII. El sitio de Cruz Pampa cerca de Porco está asociado con una unidad doméstica ocupada por un español o persona con herencia indígena, quien fue capaz de adquirir algunos bienes materiales de estilo europeo. La gente asociada con Cruz Pampa consumió principalmente animales de origen local. Existen unos pocos animales euroasiáticos importados para alimento y un uso mínimo de animales de origen no local para fines utilitarios. No obstante la presencia de un comercio considerable a través de Porco a comienzos del siglo XVII, existe poca evidencia sobre qué animales o sus productos fueron importados y consumidos. Si bien se favorecen las explicaciones sociales, los efectos biológicos de la alta elevación sobre los animales importados necesitan también ser considerados.

Los restos de fauna del sitio Tarapaya al norte de Potosí están asociados con un hostal y una unidad doméstica cerca de un grupo de baños termales. En contraste al patrón observado en Cruz Pampa, los residentes de Tarapaya importaron una variedad de animales incluyendo una predominancia en la variedad de fuentes de carne euroasiática, particularmente, 
ovejas, cabras y pollos. Una diversidad de pescados, incluyendo restos de especies lacustres ribereñas y marinas, están presentes en la muestra indicando un comercio bien establecido así como conexiones hacia el norte y sur de Tarapaya. El uso de animales fue de carácter ibérico con poco consumo de fuentes de carne nativa.

Dada la naturaleza del comercio en la región así como el movimiento de bienes hacia y desde la ciudad y las comunidades mineras vecinas, las diferencias en el uso de los animales no parecen haber estado principalmente relacionadas a las distintas ubicaciones geográficas de los dos sitios. La diferencia étnica y de estatus es interpretada como la influencia más fuerte en el uso de los animales comestibles observada en los dos sitios. Mientras estos dos ejemplos son confiables en términos de tamaño de muestra y procedencia de depósitos no disturbados, estas interpretaciones son preliminares. Se necesitan de excavaciones adicionales en un gran rango de contextos para determinar variaciones étnicas, de estatus y geográficas en el uso de los animales durante el período colonial. La muestra de Tarapaya proporciona gran sustento para las fuentes históricas indicando que el comercio permitió la importación de alimentos y animales para el centro minero. A pesar que Porco fue un componente integral de la economía minera y conectó el comercio regional e internacional involucrando la importación y exportación de productos, existen pocos indicadores que el comercio de animales comestibles fue importante o, posiblemente, deseado. Sin embargo, las excavaciones en otros contextos domésticos y comerciales son necesarias para identificar el rango de uso de los animales en el Potosí colonial y áreas vecinas. Las siguientes pautas podrían ser fructíferas vías para investigaciones futuras: (1) datos de fauna de contextos domésticos dentro de Potosí, posiblemente procedentes de varias unidades domésticas que representen gente de diferente estatus, (2) un examen de los restos de fauna para determinar si existen diferencias étnicas en la forma como los animales son procesados, (3) el examen de los restos zooarqueológicos junto con los bienes materiales a través de la preparación y servicio de los alimentos, así como con los restos de plantas a fin de identificar el origen de la "cocina andina".

Agradecimientos: Deseo agradecer a Mary Van Buren por permitir que las muestras de Porco y Tarapaya estén disponibles para su estudio, proporcionando los datos de las excavaciones y material cultural del sitio, así como por proporcionar sus comentarios para este artículo. Agradezco también a las Dras. Elizabeth Wing y Kitty Emery por su espacio de investigación en el Laboratorio de Arqueología Ambiental en el Museo de Historia Natural de Florida. Doug Nelson, del Museo de Zoología de la Universidad de Michigan, me prestó especímenes de sábalo (Prochilodus lineatus) para la identificación de algunos de los restos óseos de pescados de Tarapaya. Yo identifiqué algunas especies de aves usando la colección osteológica de aves de la División de Ornitología del Museo de Historia Natural de Florida. Augusto OyuelaCaycedo me ayudó con algunas traducciones. Y quisiera agradecer a los evaluadores y editores por sus comentarios y sugerencias. Yo me responsabilizo de cualquier error, omisión e inconsistencia que pueda surgir.

\section{Referencias Citadas}

Acarete du Biscay, M. 1968 [1698]. An Account of a Voyage up the River de la Plata and Thence Over Land to Peru. North Haven, Institute Publishing Company.

Acosta de, J. 2002 [1590]. Historia Natural y Moral de las Indias. Editado por J.E. Mangan. Duke University Press, Durham.

Arzáns de Orsúa y Vela, B. 1965 [1787]. Historia de la Villa Imperial de Potosí. Editado por L. Hanke y G. Mendoza L. Brown University Press, Providence.

Bakewell, P. 1988. Silver and Entrepreneurship in SeventeenthCentury Potosí: The Life and Times of Antonio López de Quiroga. University of New Mexico Press, Albuquerque.

Cieza de León, P. 1964 [1553]. Travels of Pedro de Cieza de León, A.D. 1532-50. Traducido por C.R. Markham. Burt Franklin, New York.
Cobb, G. 1949. Supply and transportation for the Potosí mines, 1545-1640. Hispanic American Historical Review 29:25-45.

Deagan, K. 1974. Sex, Status, and Role in the Mestizaje of Spanish Colonial Florida. Doctoral Dissertation, Department of Anthropology, University of Florida, Gainesville.

deFrance, S.D. 1993. Ecological Imperialism in the South-Central Andes: Faunal Data from Spanish Colonial Settlements in the Moquegua and Torata Valleys. Doctoral Dissertation, Department of Anthropology, University of Florida, Gainesville.

_ _ - 1996. Iberian foodways in the Moquegua and Torata Valleys of Southern Peru. Historical Archaeology 30:20-48.

- - - 2003. Diet and provisioning in the high Andes: A Spanish colonial settlement on the outskirts of Potosí, Bolivia. International Journal of Historical Archaeology 7:99-125. 
- - - 2010. Paleopathology and health of native and introduced animals on southern Peruvian and Bolivian Spanish colonial sites. The International Journal of Osteoarchaeology 20:508-524.

Eighmy, J.L., S.N. Lengyel y M. Van Buren 2006. Archaeomagnetic research in the Andean highlands: Results from the Proyecto Arqueológico Porco-Potosí, Bolivia. Ponencia presentada en Archaeological Sciences of the Americas Symposium, Tucson.

Funari, P.P., M. Hall y S. Jones 1999. Historical Archaeology: Back from the Edge. Routledge, London.

Funari, P.P., A. Zarankin y E. Stovel 2005. Global Archaeological Theory: Contextual Voice and Contemporary Thoughts. Kluwer Academic/Plenum Publishers, New York.

Hall, M. y S.W. Silliman (eds.) 2006. Historical Archaeology. Blackwell Publishing, Malden.

Jiménez de la Espada, M. 1965 [1603]. Relaciones Geográficas de Indias-Perú, II. Biblioteca de Autores Españoles, Vol. 183, Ediciones Atlas, Madrid.
Lockhart, J. 1994. Spanish Peru 1532-1560: A Social History. University of Wisconsin Press, Madison.

Presta, A.M. 1992. Juan Ortiz de Zárate, an Entrepreneur of Sixteenth-Century La Plata, Charcas. Master's Thesis, Department of History, The Ohio State University, Buenos Aires.

Reitz, E.J. 1986. Vertebrate faunal remains from Locus 19, Puerto Real, Haiti. Journal of Field Archaeology 13:317-328. 1992. Vertebrate fauna from seventeenth-century St. Augustine. Southeastern Archaeology 11:79-94.

Reitz, E.J. y B.G. McEwan 1995. Animals and the Spanish iet at Puerto Real. The Archaeology of a 16th Century Townsite on Hispaniola. Editado por K.A. Deagan. University Presses of Florida, Gainesville.

Van Buren, M. 1999. Tarapaya: An elite Spanish residence near colonial Potosí in comparative perspective. Historical Archaeology 33:101-115. 